\title{
Visual cortex in dementia with Lewy bodies: magnetic resonance imaging study
}

John-Paul Taylor, Michael J. Firbank, Jiabao He, Nicola Barnett, Sarah Pearce, Anthea Livingstone, Quoc Vuong, lan G. McKeith and John T. O'Brien

\section{Background}

Visual hallucinations and visuoperceptual deficits are common in dementia with Lewy bodies, suggesting that cortical visual function may be abnormal.

\begin{abstract}
Aims
To investigate: (1) cortical visual function using functional magnetic resonance imaging (fMRI); and (2) the nature and severity of perfusion deficits in visual areas using arterial spin labelling (ASL)-MRI.
\end{abstract}

\section{Method}

In total, 17 participants with dementia with Lewy bodies (DLB group) and 19 similarly aged controls were presented with simple visual stimuli (checkerboard, moving dots, and objects) during fMRI and subsequently underwent ASL-MRI (DLB group $n=15$, control group $n=19$ ).

\section{Results}

Functional activations were evident in visual areas in both the DLB and control groups in response to checkerboard and objects stimuli but reduced visual area V5/MT (middle temporal) activation occurred in the DLB group in response to motion stimuli. Posterior cortical perfusion deficits occurred in the DLB group, particularly in higher visual areas.

\section{Conclusions}

Higher visual areas, particularly occipito-parietal, appear abnormal in dementia with Lewy bodies, while there is a preservation of function in lower visual areas (V1 and $\mathrm{V} 2 / 3)$.

\section{Declaration of interest}

None.
In comparison to Alzheimer's dementia, individuals with dementia with Lewy bodies are disproportionately affected by visuoperceptual impairments and up to $80 \%$ experience recurrent complex visual hallucinations. ${ }^{1}$ These clinical and neuropsychological features strongly suggest that the visual system in dementia with Lewy bodies is dysfunctional, although precisely which components are affected and how remains to be clarified. Single photon emission computerised tomography (SPECT) and fluorodeoxyglucose positron emission tomography (PET) studies in dementia with Lewy bodies have demonstrated occipital and occipito-parietal visual association area hypoperfusion and hypometabolism respectively. ${ }^{2-5}$ However, occipital deficits are not consistently reported in dementia with Lewy bodies. ${ }^{3}$

Functional magnetic resonance imaging (fMRI) has been used to examine cortical visual function, although there has been only one study to date in patients with dementia with Lewy bodies, which examined cortical activation and deactivation patterns compared with patients with Alzheimer's disease using a number of visual tasks. ${ }^{6}$ However, we are not aware of studies investigating deficits in lower visual processing in dementia with Lewy bodies. There is a larger literature looking at cortical visual function in Parkinson's disease, ${ }^{7-9}$ although cortical visual activation patterns vary considerably depending on the study paradigm. Overall, there remains a need to understand the functional integrity of the cortical visual system in dementia with Lewy bodies. We sought to address this issue using the application of fMRI and arterial spin labelling (ASL)-MRI to dementia with Lewy bodies. Our specific hypotheses were:

1 that individuals with dementia would show evidence of deficits in higher visual areas on blood oxygen-level dependent (BOLD) response to visual stimuli compared with controls, but lower visual areas would show normal function;

2 that using ASL-MRI we would find posterior brain hypoperfusion at rest in individuals with dementia compared with controls; however, in light of increasing evidence for intact lower visual areas in dementia with Lewy bodies, we expected perfusion deficits to be less marked here than in higher areas.

\section{Method}

\section{Participants}

Participants with dementia with Lewy bodies (DLB group) were recruited from a local community-dwelling population of individuals who had been referred to geographically based old age psychiatry and neurology services. The control group was selected from friends and spouses of participants included in this and previous studies. The study was approved by the local ethics committee.

Diagnosis of probable dementia with Lewy bodies was made independently by two experienced senior clinicians using the revised International Consensus Guidelines for dementia with Lewy bodies. ${ }^{10}$ Cognitive function was tested using the Cambridge Cognitive Examination ${ }^{11}$ (CAMCOG, maximum score 105) and the Mini-Mental State Examination ${ }^{12}$ (MMSE, maximum score 30). The presence and severity of any extrapyramidal signs were graded using the motor component of the Unified Parkinson's Disease Rating Scale (UPDRS). ${ }^{13}$

Control participants included in the study demonstrated no evidence of dementia (from history and score $>80$ on CAMCOG). Exclusion criteria for all participants included contraindications for MRI, severe visual impairment, previous history of alcohol or substance misuse, significant neurological or psychiatric history, focal brain lesions on brain imaging or the presence of other severe or unstable medical illness.

All participants had measurement of their best near visual acuity on Landolt broken rings or Snellen chart (test distance $40 \mathrm{~cm}$ ) after correction of any refractive errors. Assessment of visuoperceptual function was carried out using previously described angle discrimination and overlapping figures tasks, with 
20 trials administered for each task. ${ }^{14}$ Prior to MRI in the DLB group, cognitive fluctuations were assessed using the Clinician Assessment of Fluctuation (CAF) scale and the One Day Fluctuation Assessment Scale (ODFAS). ${ }^{15}$ For assessment of visual hallucinations, caregivers were asked to complete the hallucinations subscale of the Neuropsychiatric Inventory $\left(\mathrm{NPI}^{\text {hall }}\right)$, which enquires about the occurrence of visual hallucinations in the past month. The NPI ${ }^{\text {hall }}$ score (frequency $x$ severity) was used in analyses to determine the association of the severity and frequency of visual hallucinations with imaging indices.

\section{Neuroimaging data acquisition}

Participants were scanned on a 3T whole body MRI scanner (Achieva scanner; Philips Medical System, The Netherlands). Images acquired included a standard whole brain structural scan (three-dimensional (3D) MPRAGE, sagittal acquisition, slice thickness $1.2 \mathrm{~mm}$, in plane resolution $0.94 \times 0.94 \mathrm{~mm}$; repetition time (TR) $9.6 \mathrm{~ms}$; echo time (TE) $4.6 \mathrm{~ms}$; flip angle $8^{\circ}$; SENSE factor 2). Functional MRI data were collected with a gradient-echo echo planar imaging $(\mathrm{EPI})$ sequence $(\mathrm{TR}=1.92 \mathrm{~s} ; \mathrm{TE}=40 \mathrm{~ms}$; field of view (FOV) $192 \times 192 \mathrm{~mm}^{2}$; matrix size $64 \times 64$, flip angle $90^{\circ}$, 27 slices, slice thickness $3 \mathrm{~mm}$, slice gap $1 \mathrm{~mm}$ ). The length of each functional run varied between the different stimulus conditions (see below): 100 volumes (192s) were acquired for the checkerboard stimuli; 183 volumes (351 s) were acquired for the moving dot stimuli; and 234 volumes $(449 \mathrm{~s})$ were acquired for objects stimuli.

Subsequently, within the same scanning session, resting cerebral perfusion imaging data were collected with the participants instructed to close their eyes during the acquisition. Cerebral perfusion imaging used a multislice pulsed ASL sequence - FAIR (Flow sensitive Alternating Inversion Recovery ${ }^{16}$ ) - with 4 slices $(\mathrm{TE}=26 \mathrm{~ms}, \mathrm{TR}=4000 \mathrm{~ms}$, inflow time $1700 \mathrm{~ms}$, in plane resolution $4 \times 4 \mathrm{~mm}^{2}$, slice thickness $6 \mathrm{~mm}$, FOV $=256 \times 256 \mathrm{~mm}^{2}$ ) and incorporated a $10 \mathrm{~ms}$ bipolar gradient to suppress bulk flow. Two spatially contiguous acquisitions of FAIR giving 8 slices were acquired, angled at $30^{\circ}$ to the corpus callosum to cover the occipital and inferior parietal lobe.

To quantify $\mathrm{T} 1$ for use in the perfusion quantification, we used a rapid inversion recovery gradient-echo EPI sequence with: $\mathrm{TR}=15000 \mathrm{~ms} ; \mathrm{TE}=24 \mathrm{~ms} ; \mathrm{T} 1$ from $0.25 \mathrm{~s}$ to $2.5 \mathrm{~s}$ in 12 equal steps; FOV $=256 \times 208 \mathrm{~mm}$; SENSE factor 2; in plane resolution $2 \times 2 \mathrm{~mm}$; 72 slices, slice thickness $2 \mathrm{~mm}$.

\section{Functional MRI stimulus presentation}

Previously reported activation differences on BOLD fMRI between controls and the DLB group may be due in part to task performance differences. ${ }^{6}$ To limit the confounding effect of performance (either over- or underactivity) so that we could specifically test for (any) differences in visual cortical processing, our participants passively viewed simple visual stimuli rather than carrying out an active behavioural task. Visual stimuli were backprojected onto a screen at the foot of the scanner and individuals viewed the stimuli via a mirror positioned above their eyes. Functional MRI compatible goggles with lenses that ranged from -4.0 to 4.0 diopters ( 0.5 increment) were used to correct any refractive errors.

Participants were asked to focus on a central cross-hair set against a grey background. The stimulus presentation was controlled by the psychophysics toolbox (http://psychtoolbox. org/; extension for Matlab (Mathworks, Natick, Massachusetts, USA). ${ }^{17}$
A block design was used for three different stimulus conditions: flashing checkerboards (checkerboard stimulus), pictures of objects (objects stimulus), and moving dot fields (motion stimulus). The checkerboard stimulus consisted of five $19.2 \mathrm{~s}$ blocks of a flashing black-and-white checkerboard, alternating with five $19.2 \mathrm{~s}$ baseline blocks of a blank screen. The checkerboard flickered with a frequency of $7.5 \mathrm{~Hz}$. The object stimulus consisted of seven $18 \mathrm{~s}$ blocks of intact pictures of objects, alternating with seven $18 \mathrm{~s}$ blocks of images of 'scrambled' texture patterns, which had the same low-level contrast and luminance as the pictures of the objects. Between blocks, a grey screen with fixation cross was displayed. Six stimuli were presented on each block. The stimuli were shown for $1 \mathrm{~s}$, with a $2 \mathrm{~s}$ fixation cross between stimuli. For the motion stimulus, there were $189.6 \mathrm{~s}$ blocks of expanding white dots, alternating with $189.6 \mathrm{~s}$ blocks of stationary but flickering white dots. All stimuli subtended a maximum of $4^{\circ}$ of visual angle. The checkerboard stimulus was used to stimulate lower visual areas (V1-V3), the objects stimulus was used to stimulate higher visual areas in lateral-occipital cortex, ${ }^{18-20}$ and the motion stimulus was used to stimulate posterior temporal areas. ${ }^{21}$

Although participants passively viewed the visual stimuli to avoid performance confounds, to maintain their attention to the stimuli during the fMRI component of the study, a small intermittent pink dot appeared approximately every $30 \mathrm{~s}$ over the central fixation point to which participants were instructed to respond to by squeezing a response button placed in their right hand, with disappearance of the dot either on response or after $5 \mathrm{~s}$.

\section{Functional MRI analysis}

For each participant, the T1 anatomical image was segmented and spatially normalised in SPM5 (www.fil.ion.ucl.ac.uk/spm/) using the default parameters. The fMRI data for each stimulus condition were first motion corrected by aligning all functional images to the first image, and then coregistered with the T1 anatomical image. The spatial normalisation parameters from the T1 segmentation were used to write out the EPI data in standard space with a voxel size of $3 \times 3 \times 3 \mathrm{~mm}$. The normalised images were then smoothed $(6 \times 6 \times 6 \mathrm{~mm}$ full width half-maximum Gaussian kernel). A high pass filter of $128 \mathrm{~s}$ was used, and serial correlations were removed with SPM's AR(1) model.

The general linear model (GLM) in SPM was used to conduct a whole brain analysis of the fMRI data. For each stimulus condition, we created a design matrix by convolving the time course of the two block types (i.e. flashing checkerboard and blank; objects and scrambled images; and moving dots and static flashing dots), with the canonical haemodynamic response function (HRF) and its first derivative. The six parameters from the motion correction for each functional run were included in the design matrix as covariates of no interest. The regressors were fitted to the fMRI data to produce beta estimates for each regressor. Individual participant and second-level (random effects) group analyses were conducted making comparisons between groups. Contrast images were generated from beta estimates for the following comparisons: checkerboard $v$. baseline (checkerboard stimulus contrast), moving dots $v$. static dots (motion stimulus contrast), and objects $v$. scrambled images (objects stimulus contrast). Results are shown thresholded at $P<0.05$ family wise error corrected for multiple comparisons at the voxel level and we report clusters only $\geqslant 20$ voxels in size. We used the SPM anatomy toolbox (www2.fz-juelich.de/inm/ inm-1/spm_anatomy_toolbox) to determine the location of each cluster. 
To maximise sensitivity, we also performed a region of interest (ROI) analysis focusing on the visual areas. For fMRI data, five ROIs in Montreal Neurological Institute (MNI) space were defined averaging across left and right hemispheres. Four ROIs (V5/MT (middle temporal area); V1; V2 and V3 combined; and V4) were taken from the SPM anatomy toolbox. A fifth area (lateral occipital cortex, LOC) was a $1 \mathrm{~cm}$ diameter sphere (20 voxels in volume) centred on the average of coordinates described in a number of previously published reports (online Fig. DS1). ${ }^{18-20}$ For each participant, the magnitude of activation for the different conditions in each ROI was determined by averaging the relevant beta parameter from each participant's fitted beta estimates from the general linear model.

\section{Cerebral perfusion analysis}

T1 was determined by fitting the inversion recovery images with a relaxation curve on a pixel by pixel basis. Motion correction was applied to each ASL image-set. The ASL images and the T1 map were co-registered with the 3D-T1 anatomical scan. Each ASL scan was split into 'control' and 'tag' image series. Each series was then averaged and the difference image $\mathrm{dM}$ was created by subtracting the mean control image from the mean tag images, while the magnitude image $\mathrm{M}$ was calculated by taking the average of all ASL images. Perfusion values were then calculated on a voxel-wise basis from the $\mathrm{dM}, \mathrm{M}$ and $\mathrm{T} 1$ images, using a value of $1.932 \mathrm{~s}$ for $\mathrm{T} 1$ of blood, and $0.9 \mathrm{ml} / \mathrm{g}$ for the blood-brain partition coefficient. $^{22}$

The calculated perfusion images were then spatially normalised, using the parameters from the 3D-T1 scan. Since we had limited spatial coverage, a mask in MNI space was created of voxels which had ASL data from all participants. As well as using the ROIs from fMRI analysis (V1, V2/3, V4, V5/MT and LOC), we derived a further two from the Harvard Oxford atlas (in FSL, www.fmrib.ox.ac.uk/fsl) encompassing the precuneus and the superior lateral occipital (SLO) region because of the previously reported widespread posterior perfusion found in dementia with Lewy bodies in these areas. ${ }^{23,24}$ We calculated mean perfusion within these ROIs in MNI space.

\section{Statistics}

The NPI ${ }^{\text {hall }}$ score in the DLB group was correlated against fMRI beta values and ASL perfusion rates in the ROIs. Comparison of fMRI and perfusion data was also made using ROIs between the control and DLB groups correcting for age, even though groups were not significantly different, as this has been suggested to be a significant factor leading to region-specific decreases and increases in perfusion. ${ }^{25}$ Group comparisons were made using Student $t$-test and we hypothesed that there would be no difference in ROI BOLD activations between the DLB and control groups in V1-4 to checkerboard stimulus, but reduced activations in V5/MT and LOC to motion and object visual stimuli respectively. For the ASL-MRI data, we hypothesed that ROI perfusion would be normal in V1-3 but reduced in higher visual areas. Secondary analyses were done to ensure that any notable findings were related to group differences in visual function rather than owing to other factors by examining the association between key demographic/disease factors (age, visual acuity, UPDRS motor subscale score, CAMCOG score, age at onset of dementia, duration of any dementia, CAF and medication use) and imaging ROI. Parametric and non-parametric correlations were used depending on the normality of the data. All values reported are means (s.e.) unless otherwise stated and $t$-tests were two-tailed.

\section{Results}

\section{Demographics}

We recruited 19 individuals with dementia with Lewy bodies and 19 controls; one patient with dementia was subsequently excluded on the basis that he could not tolerate lying flat in the MRI scanner. Another person with dementia failed to respond to the motor task during scanning and demonstrated no occipital lobe activation to the checkerboard stimulus and thus their data were not included in the fMRI analysis. Arterial spin labelling MRI data from two other participants with dementia were excluded from the perfusion analysis due to excessive movement artefact during ASL-MRI acquisition, although fMRI data from these individuals were available. Thus, data were available on 17 individuals with dementia for the fMRI analyses and 15 individuals with dementia for the ASL-MRI analyses.

Demographic features of the participants who completed the study are shown in Table 1. Age, gender and visual acuity were comparable between control and DLB groups with, as expected, reduced cognitive performance on CAMCOG and MMSE, lower visuoperceptual task scores and higher UPDRS scores in the DLB group compared with controls.

Table 1 Demographic, cognitive and motor characteristics of participants included in study ${ }^{a}$

\begin{tabular}{|c|c|c|c|}
\hline & $\begin{array}{l}\text { Control group } \\
\qquad(n=19)\end{array}$ & $\begin{array}{l}\text { DLB group } \\
\qquad(n=17)\end{array}$ & $P$ \\
\hline Age, years: mean (s.d.) & $77.6(7.1)$ & $81.2(5.6)$ & 0.10 \\
\hline Males: females, $n$ & $11: 8$ & $10: 8$ & 1.00 \\
\hline $\begin{array}{l}\text { UPDRS motor subscale, } \\
\text { mean (s.d.) }\end{array}$ & $0.9(1.6)$ & $33.9(14.0)$ & $<0.001$ \\
\hline MMSE, mean (s.d.) & $29.0(1.2)$ & $18.8(5.1)$ & $<0.001$ \\
\hline CAMCOG, mean (s.d.) & & & \\
\hline Total score & $96.5(3.4)$ & $64.8(14.0)$ & $<0.001$ \\
\hline Executive subscore & $22.1(3.4)$ & $10.4(4.8)$ & $<0.001$ \\
\hline Memory subscore & $23.6(2.1)$ & $15.6(4.2)$ & $<0.001$ \\
\hline $\begin{array}{l}\text { Visual acuity (decimalised), } \\
\text { mean (s.d.) }\end{array}$ & $0.64(0.29)$ & $0.55(0.26)$ & 0.32 \\
\hline $\begin{array}{l}\text { Age at onset of dementia, } \\
\text { mean (s.d.) }\end{array}$ & - & $77.4(5.7)$ & N/A \\
\hline $\begin{array}{l}\text { Duration of dementia, } \\
\text { months: mean (s.d.) }\end{array}$ & - & $45.4(32.2)$ & N/A \\
\hline CAF, mean (s.d.) & - & $8.2(4.4)$ & N/A \\
\hline ODFAS, mean (s.d.) & - & $6.2(4.7)$ & N/A \\
\hline $\mathrm{NP}^{\text {hall }}$, mean (s.d.) & - & $3.1(2.3)$ & N/A \\
\hline $\begin{array}{l}\text { Angle discrimination task } \\
\text { (score out of 20), mean (s.d.) }\end{array}$ & $19.9(0.2)$ & $15.5(5.6)$ & $<0.001$ \\
\hline $\begin{array}{l}\text { Overlapping figures task } \\
\text { (score out of 20), mean (s.d.) }\end{array}$ & $19.8(0.3)$ & $14.8(3.6)$ & $<0.001$ \\
\hline $\begin{array}{l}\text { On cholinesterase inhibitor, } \\
n(\%)\end{array}$ & - & $13(72)$ & N/A \\
\hline $\begin{array}{l}\text { On anti-Parkinson } \\
\text { medication, } n(\%)\end{array}$ & - & $5(28)$ & N/A \\
\hline $\begin{array}{l}\text { L-dopa dose equivalent, } \\
\text { mg: }{ }^{b} \text { mean (s.d.) }\end{array}$ & - & $234.4(186.6)$ & N/A \\
\hline \multicolumn{4}{|c|}{$\begin{array}{l}\text { DLB, dementia with Lewy bodies; UPDRS, Unified Parkinson's Disease Rating Scale; } \\
\text { MMSE, Mini-Mental State Examination; CAMCOG, Cambridge Cognitive Examination; } \\
\text { CAF, Clinician Assessment of Fluctuation; ODFAS, One Day Fluctuation Assessment } \\
\text { Scale; NPI }{ }^{\text {hall }} \text {, Neuropsychiatric Inventory hallucinations subscale score (frequency } \times \\
\text { severity of hallucinations). } \\
\text { a. For comparison of data in control group } v \text {. DLB group, independent } t \text {-tests } \\
\text { were used for continuous data and Pearson chi-squared for categorical data. } \\
\text { b. Calculated equivalent L-dopa dose for those in the DLB group on dopaminergic } \\
\text { medication. } \\
\text { Results in bold are significant. }\end{array}$} \\
\hline
\end{tabular}




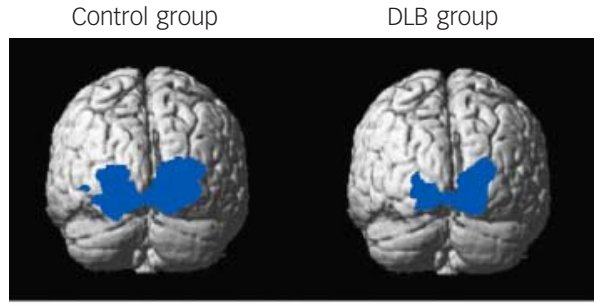

Checkerboard

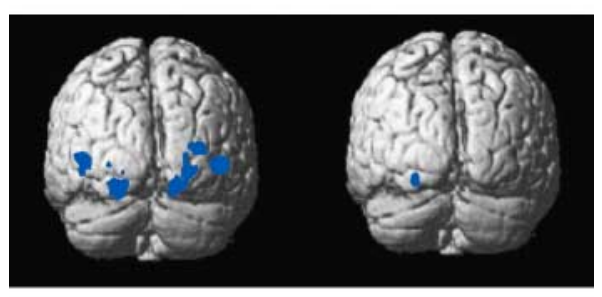

Motion

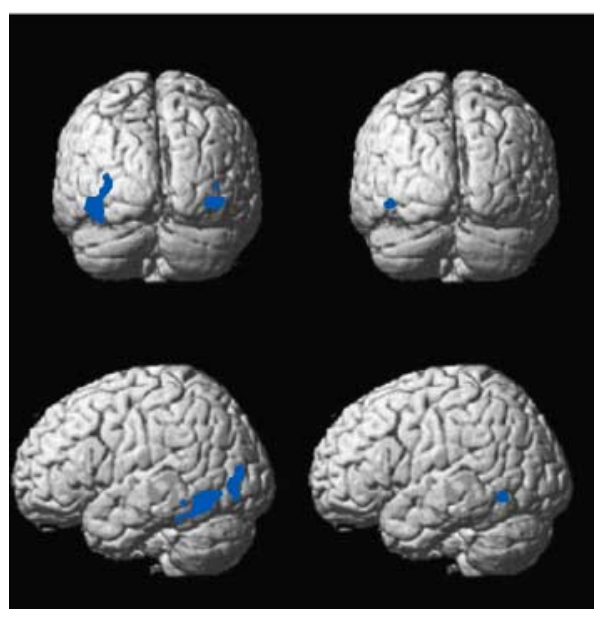

Objects

Fig. 1 Group activation maps for checkerboard, motion and objects stimuli. Blue areas indicate significant BOLD activation. See online Fig. DS2 for graduated colour activation maps.

Thresholded at $P<0.05$ family wise error corrected and superimposed on average brain template. Contrasts include checkerboard $v$. static image (checkerboard stimulus), moving dots $v$. static image (motion stimulus) and objects $v$. scrambled image (objects stimulus). BOLD, blood oxygen-level dependent; DLB, dementia with Lewy bodies.

\section{Functional MRI activations}

Distinct and overlapping networks $(P<0.05$ family wise error corrected) were evident for each of the visual stimuli for both control and DLB groups (Fig. 1 and Tables $2-4$ and online Fig. DS2). Significant cluster activations for checkerboard stimulus lay within V1 and V2 for both control and DLB groups, with controls in addition showing significant clusters over V3. For the motion stimulus contrast, both DLB and control groups had significant activations in V3 and V4, with activation in V5/MT only present in the control group. The objects stimulus contrast demonstrated activation clusters in controls overlying the left inferior temporal gyrus, left fusiform gyrus, left inferior and middle occipital gyrus. No clusters $\geqslant 20$ voxels were noted in participants with dementia with this stimulus.

Whole brain analyses comparing groups, however, did not demonstrate any significant differences in activation between DLB and control groups for each of the tasks. Region of interest analysis (Fig. 2) for lower visual areas confirmed this finding, with no differences in activity between the control and DLB groups for checkerboard stimulus (V1 ROI) or objects stimulus analyses (LOC ROI). However, the ROI localised on the V5/MT showed that the DLB group had reduced activation to the motion stimulus in this area compared with controls $(P=0.02)$. The results of the exploratory analyses of BOLD activity in the other ROIs for each of the tasks are shown in online Table DS1. Of note, there were no significant differences between DLB and control groups in the BOLD response to the three visual stimuli in V1, V2/3 or V4.

\section{Cerebral perfusion}

Cerebral perfusion within the segment of brain covered by the FAIR image acquisition was significantly reduced in the DLB group compared with controls within inferior parietal and posterior cingulate/precuneus (Fig. 3 and Table 5 and online Fig. DS3). Lower visual areas (V1-3) appeared relatively intact.

Perfusion deficits in dementia compared with controls were confirmed in ROI analysis (Fig. 4). Correcting for age, the reduction was not significant in $\mathrm{V} 1(P=0.25)$ or $\mathrm{V} 2 / 3(P=0.19)$, but approached significance in V4 $(P=0.08)$. The reduction was

\begin{tabular}{|c|c|c|c|c|c|}
\hline Cluster & $P$ corrected & Size (voxels) & Anatomical loci & Maximum Z & Maximum Z position (MNI) \\
\hline Control group & $<0.001$ & 899 & $\begin{array}{l}\text { Right V1 } \\
\text { Right area V2 } \\
\text { Left V3 } \\
\text { Left V2 }\end{array}$ & 6.80 & $9,-93,-9$ \\
\hline DLB group & $<0.001$ & 541 & $\begin{array}{l}\text { Right V1 } \\
\text { Right V2 } \\
\text { Left V2 } \\
\text { Left V1 }\end{array}$ & 6.82 & $18,-93,0$ \\
\hline
\end{tabular}

BOLD, blood oxygen-level dependent; MNI, Montreal Neurological Institute.

a. Significant clusters in Statistical Parametric Mapping analysis, local maxima more than $4 \mathrm{~mm}$ apart. Data presented were all significant at the $P<0.05$ family wise error corrected level.

\begin{tabular}{|c|c|c|c|c|c|}
\hline Cluster & $P$ corrected & Size (voxels) & Anatomical loci & Maximum Z & Maximum Z position (MNI) \\
\hline \multicolumn{6}{|c|}{ Control group } \\
\hline 1 & $<0.001$ & 27 & $\begin{array}{l}\text { Left V4 } \\
\text { Left V3 }\end{array}$ & 5.73 & $-21,-84,-18$ \\
\hline 2 & $<0.001$ & 21 & $\begin{array}{l}\text { Right V2 } \\
\text { Right V3 }\end{array}$ & 5.44 & $18,-84,-15$ \\
\hline 4 & $<0.001$ & 27 & Left V5 & 5.39 & $-42,-69,3$ \\
\hline 5 & $<0.001$ & 34 & Right V5 & 5.24 & $45,-66,0$ \\
\hline
\end{tabular}




\begin{tabular}{|c|c|c|c|c|c|}
\hline Cluster & $P$ corrected & Size (voxels) & Anatomical loci & Maximum Z & Maximum Z position (MNI) \\
\hline \multicolumn{6}{|c|}{ Control group } \\
\hline \multirow[t]{3}{*}{1} & $<0.001$ & 81 & Left inferior temporal gyrus & 6.58 & $-42,-60,-9$ \\
\hline & & & Left fusiform gyrus & 5.69 & $-36,-48,-21$ \\
\hline & & & Left inferior occipital gyrus & 6.49 & $-39,-63,-6$ \\
\hline 2 & $<0.001$ & 24 & Left middle occipital gyrus & 5.40 & $-36,-78,0$ \\
\hline
\end{tabular}

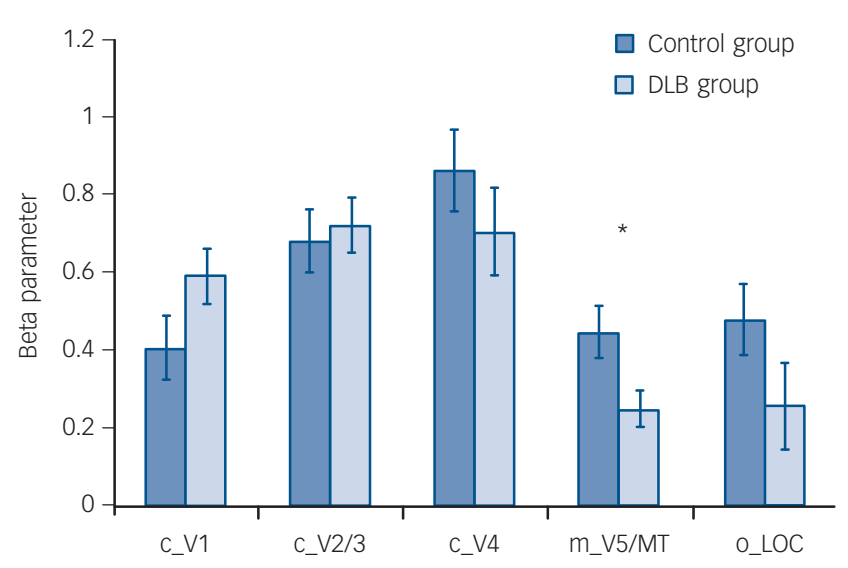

Fig. 2 Bar chart showing region of interest (ROI) analysis comparing control group $v$. dementia with Lewy bodies (DLB) group with regard to functional BOLD activations to different visual stimuli (mean (s.e.)).

C_V1, checkerboard activation in V1 ROl; C_V2/3, checkerboard activation in V2/3 ROI; C_V4, checkerboard activation in V4 ROI; m_V5/MT, motion activation in ROI V5/MT; O_LOC, objects activation in ROI lateral occipital complex; BOLD, blood oxygen-level dependent. There were no significant differences between groups for ROI activations except for motion stimulus contrast $(P=0.02)$. ${ }^{*} P<0.05$.

significant in: V5/MT, $P=0.05 ;$ LOC, $P=0.05$; precuneus, $P<0.001$; SLO, $P=0.004$.

\section{Associations between clinical variables and imaging changes in participants with dementia}

There were no significant correlations between visual hallucinations $\left(\mathrm{NPI}^{\text {hall }}\right)$ score and BOLD activations, although there was a negative relationship between V4 perfusion and $\mathrm{NPI}^{\text {hall }}$ ( $\mathrm{rho}=-0.52$, $P=0.04$; online Fig. DS4), but not perfusion in other ROIs. Angle discrimination task scores in dementia did not correlate significantly with either BOLD activations or perfusion. Overlapping figures task scores in dementia were, however, associated with increased perfusion in lower but not higher visual areas $(\mathrm{V} 1, \quad$ rho $=0.49, \quad P=0.05 ; \quad \mathrm{V} 2 / 3, \quad$ rho $=0.55, \quad P=0.03 ; \quad \mathrm{V} 4$, rho $=0.55, P=0.03$; online Fig. DS5).

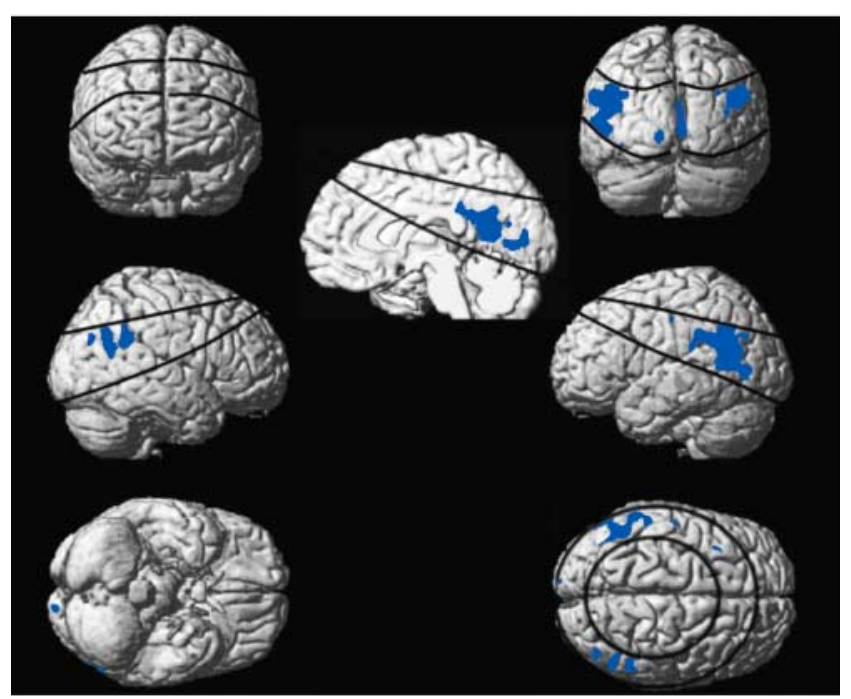

Fig. 3 Statistical Parametric Mapping t-plot showing differences in cerebral perfusion thresholded at $P<0.001$ uncorrected at group level between control and DLB groups (blue areas indicating reduced perfusion in the DLB group relative to controls) and superimposed on average brain template. Lines demarcate boundaries of overlap of perfusion slices sampled. See online Fig. DS3 for exemplar raw cerebral perfusion and graduated colour SPM perfusion maps.

There was no relationship between CAMCOG score, or visual acuity and BOLD response or perfusion in any ROI in either the control or DLB group. In the control group, participants' perfusion in ROI of SLO and LOC correlated negatively with age (SLO $r=-0.45, P=0.05$; LOC $r=-0.58, P=0.01$ ), although no relationship was noted in the DLB group. We found a negative relationship between UPDRS score and SLO ROI perfusion (rho = $-0.66, P=0.006)$ in participants with dementia, although there was no association between UPDRS scores and other ROIs for perfusion or functional BOLD activations. There were no significant differences in BOLD or perfusion data in participants with dementia taking or not taking cholinesterase inhibitors and on or off dopaminergic medications.

\begin{tabular}{|c|c|c|c|c|c|}
\hline Cluster & $P$ corrected & Size (voxels) & Anatomical loci & Maximum Z & Maximum Z position (MNI) \\
\hline 1 & $<0.001$ & 39 & $\begin{array}{c}\text { Right V1 } \\
\text { Right V2 } \\
\text { Right precuneus }\end{array}$ & 5.22 & $18,-54,12$ \\
\hline 2 & $<0.001$ & 20 & $\begin{array}{l}\text { Left inferior parietal cortex } \\
\text { Left supramarginal gyrus }\end{array}$ & 5.11 & $-45,-54,21$ \\
\hline
\end{tabular}




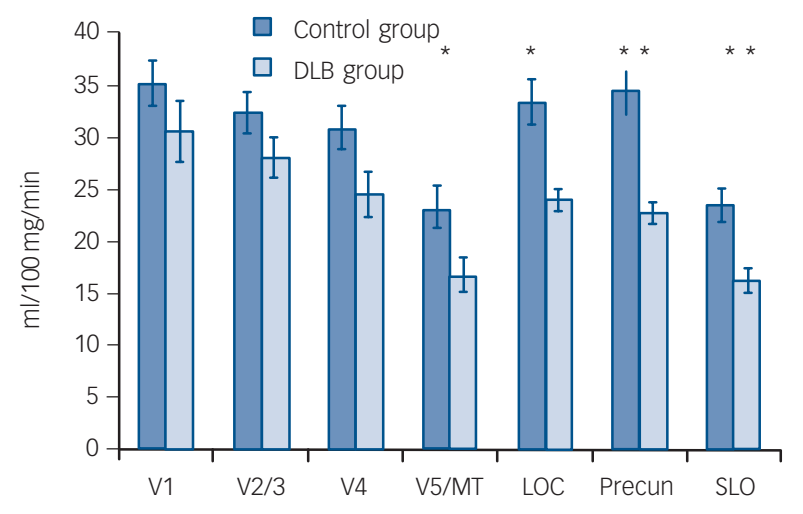

Fig. 4 Bar chart showing region of interest (ROI) analysis comparing control $v$. dementia with Lewy bodies (DLB) group with regard to perfusion (mean (s.e.)).

LOC, lateral occipital complex; Precun, precuneus; SLO, superior lateral occipital region. Mean per cent reduction in perfusion: V1, $13.1 \%$; V2-3, 13.4\%; V4, 20.5\%; V5/MT, 27.8\%; LOC 28.2\%; Precun, 33.7\%; SLO, 28.2\%. ${ }^{*} P<0.05$; ${ }^{* * P} P<0.01$.

\section{Discussion}

\section{Functional activations to passively viewed visual stimuli}

We found no significant differences in fMRI response between the control and DLB groups to checkerboard, objects or motion stimuli in V1 and V2/V3. These findings are comparable to the one other fMRI study in dementia with Lewy bodies by Sauer et $a l .^{6}$ Although these researchers did not specifically examine striate visual cortical activation to their visual stimuli, it was notable that their colour task led to similar V4 activity between participants with dementia and controls. Thus overall, given that BOLD activity is presumed to correlate with neuronal activity, ${ }^{26}$ the similar levels of activation to simple visual stimuli in the control and DLB groups imply that, in lower visual areas at least, visual processing function in dementia with Lewy bodies is not grossly perturbed. This conclusion is reinforced by the lack of specific pathological change evident in the occipital lobe of individuals with dementia with Lewy bodies at the macrostructural ${ }^{27-29}$ and microstructural level, ${ }^{30}$ comparable levels of visual cortical excitability in dementia compared with controls ${ }^{31}$ as well as our ASL findings (see below) of a non-significant reduction in lower visual area perfusion.

We found reduced activation in the lateral occipito-temporal cortex (V5/MT) on ROI analysis to the motion stimulus, which is consistent with previous evidence that individuals with Parkinson's disease or dementia with Lewy bodies who hallucinate show visual motion deficits both on behavioural testing ${ }^{14}$ and on functional imaging. ${ }^{6,7}$ However, it is notable that no significant differences were noted on the motion task on whole brain analyses. Such analyses may be unduly conservative when testing a priori hypotheses but, nonetheless, our findings should be considered preliminary and require replication.

The potential finding of reduced activity in V5/MT to motion stimuli, together with lack of group difference in LOC response to objects stimuli are somewhat at odds with neuropathological findings, although there have been very few investigations of visual pathway pathology in dementia with Lewy bodies; ${ }^{30,32}$ Lewy body pathology in the inferior temporal lobe has been linked to visual hallucinations. In contrast, Lewy body deposition in occipital and parietal areas is low. Nevertheless, perfusion (see below and
Colloby et $a l^{23}$ and Fong et $a l^{24}$ ) and metabolism deficits ${ }^{5}$ have been observed in both occipito-parietal and occipito-temporal areas. It may be the interaction between dysfunctional occipitotemporal and occipito-parietal processing streams or indeed disruption to visual white matter connectivity ${ }^{33}$ that contributes to the visuoperception difficulties and visual hallucinations seen in dementia with Lewy bodies.

\section{Perfusion deficits in dementia with Lewy bodies}

The finding of reduced posterior perfusion using ASL-MRI is consistent with prior evidence of hypoperfusion/hypometabolism in dementia with Lewy bodies. However, the extent to which the occipital lobe is affected remains controversial, with Kemp et $a l^{3}$ showing a lack of involvement, and others occipital reductions. ${ }^{2,34}$ In part, this disparity may be driven by differences in severity and stage of illness of the dementia cohorts involved in the different studies. ${ }^{24}$

Dopaminergic changes may also be relevant, as we found a negative correlation between the severity of Parkinsonism as measured on the UPDRS and perfusion in SLO ROI. A study with PET in patients with Parkinson's disease ${ }^{35}$ noted a relationship between occipital hypometabolism and nigrostriatial dysfunction, although more recent findings using ${ }^{123} \mathrm{I}$-FP-CIT/SPECT and ECD/SPECT in Parkinson's disease with dementia and patients with dementia with Lewy bodies ${ }^{36}$ failed to find any link between regional cerebral perfusion and nigrostriatal loss. This combined with a lack of any observed effect of dopaminergic medication on perfusion in the present study indicate that the role of dopaminergic mediated processes on visual function in dementia with Lewy bodies is more complex and may depend on other factors, for example, the severity of co-associated cortical neurodegeneration and/or concurrent changes in other neurotransmitter systems. $^{37}$

\section{Imaging findings related to visual hallucinations, visuoperceptual function and cholinesterase inhibitors}

There are no comparative fMRI studies in people with dementia with Lewy bodies examining the relationship between BOLD response and severity of hallucinations. There is a more extensive literature in Parkinson's disease, where studies have demonstrated widely distributed changes in activation patterns (ranging from the lower visual system, through to visual association areas and frontal areas) in people who hallucinate compared with those who do not, but there is no established or consistent pattern to these (see e.g. Stebbins et al, ${ }^{7}$ Ramírez-Ruiz et $a l^{8}$ and Meppelink et $\left.a l^{9}\right)$.

In our study we found no evidence of any association between the severity and frequency of visual hallucinations and BOLD activations to any of our visual task probes. The disparity of this finding compared with the Parkinson's disease data may be as a result of intrinsic differences between our study and those in people with Parkinson's disease in terms of disease process (dementia with Lewy bodies $v$. Parkinson's diease), cognitive function (dementia $v$. Parkinson's disease without dementia), dopaminergic/nigrostriatal deficiency, and lack of a significant non-hallucinator group in our study.

However, we did note an association between the severity of visual hallucinations and the degree of hypoperfusion deficits in V4. Visual area V4 is important for colour ${ }^{38}$ and is an intermediate module within the ventral visual stream for object and form processing ${ }^{39}$ and thus hypoperfusion of this region in association with visual hallucinations severity is certainly 
plausible. However, we did not see any association between hypoperfusion in higher visual areas such as the inferior temporal cortex, and Lewy body pathology in this area has been associated with visual hallucination occurrence. ${ }^{32}$ Nevertheless, this and other studies in dementia with Lewy bodies and Parkinson's disease which have demonstrated coupling between visual hallucinations and posterior perfusion deficits have focused on comparing people who hallucinate with people who do not, ${ }^{7-9,40}$ whereas in the present sample all participants with dementia with Lewy bodies hallucinated to varying degrees. Thus, perfusion deficits may be a marker of a neural environment that is permissive to the manifestation of visual hallucinations, but this does not indicate they are causally related.

Angle discrimination task and overlapping figures task are differentially impaired in dementia with Lewy bodies compared with other visuoperceptive tasks. ${ }^{14}$ Although the neurobiological basis of both tasks remains unclear, it is likely that the angle task is more dependent on the occipito-parietal or dorsal visual stream, ${ }^{41}$ and that the overlapping figures task may involve activity within the occipito-temporal ventral stream, ${ }^{42}$ given that this latter task requires object recognition. In the present study we did not see any association between the angle or overlapping figures task with BOLD activation to visual stimuli. This may suggest that the neural substrate that is responsive to the visual stimuli does not overlap the same structures responsible for the performance in the visuoperceptive tasks chosen in this study. However, there was an association between greater perfusion in lower visual areas (V1-4) in dementia with Lewy bodies and overlapping figures task scores, which may indicate that the integrity of lower visual areas is important in dementia with Lewy bodies for the maintenance of visuoperceptual function associated with the ventral visual stream.

There were no differences in either BOLD task-related activations or perfusion in the DLB group on or off cholinesterase inhibitors. Previous reports have demonstrated increased occipital perfusion in individuals treated with donepezil, a cholinesterase inhibitor. $^{24,43}$ The present study was cross-sectional and thus we cannot exclude the possibility that cholinesterase inhibitor use in individuals may have modulated the imaging findings.

\section{Clinical implications}

Our results suggest that lower visual areas are functionally relatively intact and respond normally to simple visual stimuli. Abnormalities in the visual system do appear to emerge at higher levels and these may contribute towards visuoperceptive dysfunction and visual hallucinations, which are common in dementia with Lewy bodies. However, whether these abnormalities include both the ventral and dorsal visual stream or are a product of an interaction between the two remains to be resolved, and certainly the integrity of lower visual areas may also be important for maintaining certain aspects of visuoperceptual function. Our understanding of the role of functional synaptic and neurochemical changes in these lower areas also remains deficient. For example, O'Brien $e t a l^{37}$ found a relationship between nicotinic receptor binding in primary and secondary visual cortices and visual hallucinations in individuals with dementia with Lewy bodies, which were not associated with occipital perfusion deficits, suggesting that significant functional receptor changes can occur in the absence of obvious alterations in perfusion. Further work combining investigative modalities in individuals with dementia with Lewy bodies that examine regional receptor changes, perfusion/metabolism and functional responsivity to visual stimuli and their relationship with observed clinical visual symptomatology are needed.

\section{Funding}

This work was supported by the UK NIHR Biomedical Research Centre for Ageing and Agerelated disease award to the Newcastle upon Tyne Hospitals NHS Foundation Trust, the Newcastle Healthcare Charity and by the Academy of Medical Sciences and Wellcome Trust Starter Grants scheme for Clinical Lecturers.

John-Paul Taylor, MBBS(Hons), PhD, MRCPsych, Michael J. Firbank, PhD, Institute for Ageing and Health, Campus for Aging and Vitality; Jiabao He, PhD, Institute of Cellular Medicine and Newcastle Magnetic Resonance Centre; Nicola Barnett, BSC(Hons), Sarah Pearce, MBBS, Anthea Livingstone, MBBS, Institute for Ageing and Health, Campus for Aging and Vitality; Quoc Vuong, PhD, Institute of Neuroscience, Ian G. McKeith, MD, FRCPsych, FMedSci, John T. O'Brien, DM, FRCPsych, Institute for Ageing and Health, Campus for Aging and Vitality, Newcastle University, Newcastle upon Tyne, UK

Correspondence: Dr John-Paul Taylor, Institute for Ageing and Health Newcastle University, Wolfson Research Centre, Campus for Ageing and Vitality, Newcastle upon Tyne NE4 5PL, UK. Email: john-paul.taylor@ncl.ac.uk

First received 15 Jul 2011, final revision 17 Nov 2011, accepted 21 Dec 2011

\section{References}

1 Aarsland D, Ballard C, Larsen JP, McKeith I. A comparative study of psychiatric symptoms in dementia with Lewy bodies and Parkinson's disease with and without dementia. Int J Geriatr Psychiatry 2001; 16: 528-36.

2 Pasquier J, Michel BF, Brenot-Rossi I, Hassan-Sebbag N, Sauvan R, Gastaut JL. Value of (99m)TC-ECD SPET for the diagnosis of dementia with Lewy bodies. Eur J NuCl Med Mol Imaging 2002; 29: 1342-8.

3 Kemp PM, Hoffmann SA, Tossici-Bolt L, Fleming JS, Holmes C. Limitations of the HMPAO SPECT appearances of occipital lobe perfusion in the differential diagnosis of dementia with Lewy bodies. Nucl Med Commun 2007; 28: 451-6.

4 Lobotesis K, Fenwick JD, Phipps A, Ryman A, Swann A, Ballard C, et al. Occipital hypoperfusion on SPECT in dementia with Lewy bodies but not AD. Neurology 2001; 56: 643-9.

5 Imamura $\mathrm{T}$, Ishii $\mathrm{K}$, Hirono $\mathrm{N}$, Hashimoto $\mathrm{M}$, Tanimukai S, Kazuai $\mathrm{H}$, et al. Visual hallucinations and regional cerebral metabolism in dementia with Lewy bodies (DLB). NeuroReport 1999; 10: 1903-7.

6 Sauer J, ffytche DH, Ballard C, Brown RG, Howard R. Differences between Alzheimer's disease and dementia with Lewy bodies: an fMRI study of taskrelated brain activity. Brain 2006; 129: 1780-8.

7 Stebbins GT, Goetz CG, Carrillo MC, Bangen KJ, Turner DA, Glover GH, et al. Altered cortical visual processing in PD with hallucinations: an fMRI study. Neurology 2004; 63: 1409-16.

8 Ramírez-Ruiz B, Martí M-J, Tolosa E, Falcón C, Bargalló N, Valldeoriola F, et al. Brain response to complex visual stimuli in Parkinson's patients with hallucinations: a functional magnetic resonance imaging study. Mov Disord 2008; 23: 2335-43.

9 Meppelink AM, de Jong BM, Renken R, Leenders KL, Cornelissen FW, van Laar $\mathrm{T}$. Impaired visual processing preceding image recognition in Parkinson's disease patients with visual hallucinations. Brain 2009; 132: 2980-93.

10 McKeith IG, Dickson DW, Lowe J, Emre M, O'Brien J T, Feldman $\mathrm{H}$, et al. Diagnosis and management of dementia with Lewy bodies. Third report of the DLB consortium. Neurology 2005; 65: 1863-72.

11 Roth M, Tym E, Mountjoy CQ, Huppert FA, Hendrie H, Verma S, et al. CAMDEX. A standardised instrument for the diagnosis of mental disorder in the elderly with special reference to the early detection of dementia. Br J Psychiatry 1986; 149: 698-709.

12 Folstein MF, Folstein SE, McHugh PR. Mini-Mental State. A practical method for grading the cognitive state of patients for the clinician. J Psychiatr Res 1975; 12: 189-98.

13 Fahn S. Unified Parkinson's disease rating scale. In Recent Developments in Parkinson's Disease: 153-63. McMillan, 1987.

14 Mosimann UP, Mather G, Wesnes KA, O'Brien JT, Burn DJ, McKeith IG. Visual perception in Parkinson disease dementia and dementia with Lewy bodies. Neurology 2004; 63: 2091-6.

15 Walker MP, Ballard CG, Ayre GA, Wesnes K, Cummings JL, McKeith IG, et al. The Clinician Assessment of Fluctuation and the One Day Fluctuation Assessment Scale. Two methods to assess fluctuating confusion in dementia. Br J Psychiatry 2000; 177: 252-6.

16 Kim SG, Tsekos NV. Perfusion imaging by a flow-sensitive alternating inversion recovery (FAIR) technique: application to functional brain imaging. Magn Reson Med 1997; 37: 425-35 
17 Pelli DG. The VideoToolbox software for visual psychophysics: transforming numbers into movies. Spat Vis 1997; 10: 437-42.

18 Grill-Spector K, Malach R. fMR-adaptation: a tool for studying the functional properties of human cortical neurons. Acta Psychol (Amst) 2001; 107: 293-321.

19 Kourtzi Z, Erb M, Grodd W, Bülthoff HH. Representation of the perceived 3-D object shape in the human lateral occipital complex. Cereb Cortex 2003; 13 911-20.

20 Lerner Y, Hendler T, Ben-Bashat D, Harel M, Malach R. A hierarchical axis of object processing stages in the human visual cortex. Cereb Cortex 2001; 11 287-97.

21 Watson JDG, Myers R, Frackowiak RSJ, Hajnal JV, Woods RP, Mazziotta JC, et al. Area V5 of the human brain: evidence from a combined study using positron emission tomography and magnetic resonance imaging. Cereb Cortex 1993; 3: 79-94.

22 Silva AC, Kim S-G. Pseudo-continuous arterial spin labeling technique for measuring CBF dynamics with high temporal resolution. Magn Reson Med 1999; 42: 425-9.

23 Colloby SJ, Fenwick JD, Williams ED, Paling SM, Lobotesis K, Ballard C, et al A comparison of (99m)Tc-HMPAO SPET changes in dementia with Lewy bodies and Alzheimer's disease using statistical parametric mapping. Eur J NuCl Med Mol Imaging 2002; 29: 615-22.

24 Fong $T$, Inouye S, Dai W, Press D, Alsop D. Association cortex hypoperfusion in mild dementia with Lewy bodies: a potential indicator of cholinergic dysfunction? Brain Imaging Behav 2010; 5: 25-35.

25 Preibisch C, Sorg C, Forschler A, Grimmer T, Sax I, Wohlschlager AM, et al. Age-related cerebral perfusion changes in the parietal and temporal lobes measured by pulsed arterial spin labeling. J Magn Reson Imaging 2011; 34 1295-302.

26 Logothetis NK, Pfeuffer J. On the nature of the BOLD fMRI contrast mechanism. Magn Reson Med 2004; 22: 1517-31.

27 Beyer MK, Larsen JP, Aarsland D. Gray matter atrophy in Parkinson disease with dementia and dementia with Lewy bodies. Neurology 2007; 69: 747-54.

28 Middelkoop HA, van der Flier WM, Burton EJ, Lloyd AJ, Paling S, Barber R, et al. Dementia with Lewy bodies and $A D$ are not associated with occipital lobe atrophy on MRI. Neurology 2001; 57: 2117-20.

29 Whitwell JL, Weigand SD, Shiung MM, Boeve BF, Ferman TJ, Smith GE, et al. Focal atrophy in dementia with Lewy bodies on MRI: a distinct pattern from Alzheimer's disease. Brain 2007; 130: 708-19.

30 Gomez-Tortosa E, Newell K, Irizarry MC, Albert M, Growdon JH, Hyman BT. Clinical and quantitative pathologic correlates of dementia with Lewy bodies. Neurology 1999; 53: 1284-91.
31 Taylor J-P, Firbank M, Barnett N, Pearce S, Livingstone A, Mosimann U, et al. Visual hallucinations in dementia with Lewy bodies: transcranial magnetic stimulation study. Br J Psychiatry 2011; 199: 492-500.

32 Harding AJ, Broe GA, Halliday GM. Visual hallucinations in Lewy body disease relate to Lewy bodies in the temporal lobe. Brain 2002; 125: 391-403.

33 Ota M, Sato N, Ogawa M, Murata M, Kuno S, Kida J, et al. Degeneration of dementia with Lewy bodies measured by diffusion tensor imaging. NMR Biomed 2009; 22: 280-4.

34 Imamura T, Ishii K, Hirono N, Hashimoto M, Tanimukai S, Kazui H, et al. Occipital glucose metabolism in dementia with lewy bodies with and without Parkinsonism: a study using positron emission tomography. Dement Geriatr Cogn Disord 2001; 12: 194-7.

35 Bohnen NI, Minoshima S, Giordani B, Frey KA, Kuhl DE. Motor correlates of occipital glucose hypometabolism in Parkinson's disease without dementia. Neurology 1999; 52: 541-6.

36 Rossi C, Volterrani D, Nicoletti V, Manca G, Frosini D, Kiferle L, et al. 'Parkinson-dementia' diseases: a comparison by double tracer SPECT studies. Parkinsonism Relat Disord 2009; 15: 762-6.

37 O’Brien JT, Colloby SJ, Pakrasi S, Perry EK, Pimlott SL, Wyper DJ, et al. Nicotinic [alpha]4[beta]2 receptor binding in dementia with Lewy bodies using 123I-5IA-85380 SPECT demonstrates a link between occipital changes and visual hallucinations. Neurolmage 2008; 40: 1056-63.

38 McKeefry DJ, Zeki S. The position and topography of the human colour centre as revealed by functional magnetic resonance imaging. Brain 1997; 120: 2229-42.

39 Pasupathy A. Neural basis of shape representation in the primate brain. Prog Brain Res 2006; 154: 293-313.

40 Perneczky R, Drzezga A, Boecker H, Forstl H, Kurz A, Haussermann P. Cerebral metabolic dysfunction in patients with dementia with Lewy bodies and visual hallucinations. Dement Geriatr Cogn Disord 2008; 25: 531-8.

41 Wood JS, Firbank MJ, Mosimann UP, Taylor JP, O'Brien JT. Development of a novel fMRI compatible visual perception prototype battery to test older people with and without dementia. J Geriatr Psychiatry Neurol 2011; 24: 73-83.

42 Alegret M, Vinyes-Junqué G, Boada M, Martínez-Lage P, Cuberas G, Espinosa $A$, et al. Brain perfusion correlates of visuoperceptual deficits in mild cognitive impairment and mild Alzheimer's disease. J Alzheimer's Dis 2010; 21: 557-67.

43 Mori T, Ikeda M, Fukuhara R, Nestor PJ, Tanabe H. Correlation of visual hallucinations with occipital rCBF changes by donepezil in DLB. Neurology 2006; 66: 935-7. 